This item was submitted to Loughborough's Research Repository by the author.

Items in Figshare are protected by copyright, with all rights reserved, unless otherwise indicated.

\title{
Delay spread emulation in machine workshops with fractals for wireless communication system planning
}

PLEASE CITE THE PUBLISHED VERSION

https://doi.org/10.1016/j.phycom.2019.100732

PUBLISHER

(C) Elsevier BV

VERSION

AM (Accepted Manuscript)

PUBLISHER STATEMENT

This paper was accepted for publication in the journal Physical Communication and the definitive published version is available at https://doi.org/10.1016/j.phycom.2019.100732.

LICENCE

CC BY-NC-ND 4.0

\section{REPOSITORY RECORD}

Adegoke, Elijah, Robert Edwards, William Whittow, and Axel Bindel. 2019. "Delay Spread Emulation in Machine Workshops with Fractals for Wireless Communication System Planning”. Loughborough University. https://hdl.handle.net/2134/37965. 
This is the final author version of

E. I. Adegoke, R. M. Edwards, W. G. Whittow, and A. Bindel, "Delay spread emulation in machine workshops with fractals for wireless communication system planning," Phys. Commun., vol. In press, p. 100732, Jun. 2019.

The DOI is: $10.1016 / j . p h y c o m .2019 .100732$

The paper can be downloaded from the journal webpage via the link below:

https://doi.org/10.1016/i.phycom.2019.100732 


\title{
Delay Spread Emulation in Machine Workshops with Fractals for Wireless Communication System Planning
}

\author{
E. I. Adegoke, R. M. Edwards, William G. Whittow, Axel Bindel
}

\begin{abstract}
This paper presents a new way to estimate delay spread in machine workspaces by using fractal geometry. In this way, inventories can be created quickly and used within a ray tracing software to estimate the radio environment of machine workspaces as part of the planning process. Delay spread is an important metric in assessing the performance of wireless technologies. Predicted 5G cyber-physical systems in workplaces will require high-density use of wirelessly connected machineto-machine RF modules. In workshops, the surfaces and edges of machines, shelves, and furniture influence the multipath/power delay profile of the space. However, with the fast construction pace and high occupancy of buildings, it is impractical to characterise the location as building work progresses. Consequently, it becomes more probable that the radio communication system deployed will perform suboptimally. In this work, the Wi-Fi band was investigated. In addition, representative simulations were also carried out at millimetre wave frequencies of $28 \mathrm{GHz}$ and $60 \mathrm{GHz}$.
\end{abstract}

\section{Index Terms}

5G, Cyber-physical systems; Internet of Things; fractals; wireless propagation; delay spread.

This research was sponsored by EPSRC and HSSMI via a CASE PhD scholarship.

E. I. Adegoke is presently with WMG, University of Warwick, Coventry CV4 7AL, U.K; email:elijah.adegoke@warwick.ac.uk

R. M. Edwards and W. G. Whittow are with the 5G Research Centre, Wolfson School of Mechanical, Electrical and Manufacturing Engineering, Loughborough University, Loughborough LE11 3TU, U.K; e-mails: r.m.edwards@lboro.ac.uk; w.g.whittow@lboro.ac.uk.

Axel Bindel is with HSSMI, London, UK. email:axel.bindel@hssmi.org 


\section{INTRODUCTION}

Next generation industrial production systems in factories may require large numbers of wireless sensors and control nodes. Such systems are now referred to as cyber-physical systems (CPS). An example of the demand for CPS is the European Industry/Factory 4.0 call that aims to provide optimized factory processes and operations [1]. Both the Internet of Things (IoT) [2] and the requirements for fifth generation $(5 \mathrm{G})$ anticipate increased machine-to-machine (M2M) data over wireless.

From a radio frequency (RF) perspective, when compared to the typical spaces humans occupy, machine workshops have interesting properties due to their high proportions of conducting surfaces and sharp edges. The multipath environment is likely to be rich with high numbers of scatterers. In order to design optimal wireless communication systems, it is helpful that the channel impulse response (CIR) is well characterised. If path delays are in the range of a single bit, it may be impossible for the receiver to properly assign energy to the corresponding bits. Thus, inter-symbol interference occurs and performance is reduced. Therefore the delay spread is closely related to the bit rate. Example applications of delay spread measurements and models are in the design of rake receivers and modulation scheme parameters such as guard-time in orthogonal frequency division multiplexing (OFDM) cyclic prefixes [3]. Knowledge of the delay spread characteristics is a needed to predict the performance of receivers. [4]. However, because of their utility value, levels of occupancy and safety regimes, measurement campaigns inside workshops are not straightforward. In this paper we propose a way to use fractals, combined with ray tracing, to greatly simplify the process of obtaining delay spread data. At $2.4 \mathrm{GHz}$, our methodology was to measure the delay spread in a machine workshop using a vector network analyser (VNA) with a reference channel and validated our method and results against other studies. We then created a ray tracing model of the machine shop and compared results from the ray tracing software with our validated measured results. The ray tracing model has in it an inventory of objects based on the machines and objects in the workshop. We then replaced the inventory using artificial fractals and validated our results against both measurements and ray tracing. In this way we have a better solution to the problem of estimating delay spread in workshops that are either planned or occupied.

In applying the work to future $5 \mathrm{G}$ cyber-physical systems, we have used our technique to study delay spread at two representative frequencies of $28 \mathrm{GHz}$ and $60 \mathrm{GHz}$. Previously, wireless 


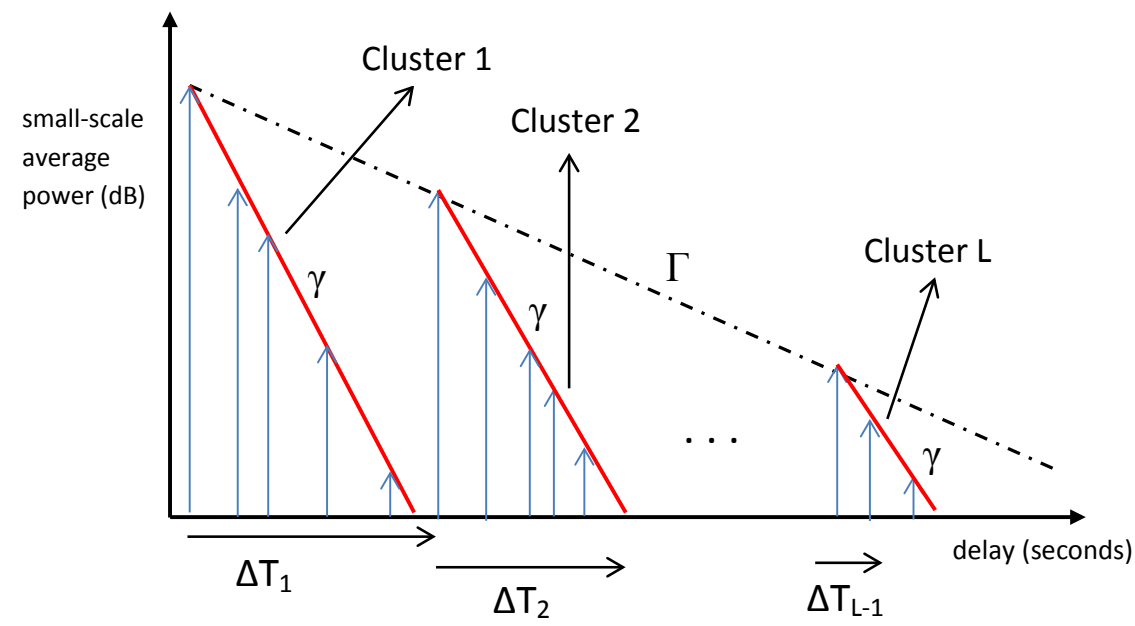

Figure 1. Saleh-Valenzuela channel impulse response model. The visually identified clusters are represented with red solid lines with corresponding decay rate $(\gamma)$, and the envelope decay constant $(\Gamma)$ is represented by the dashed solid line.

channels have been analysed using data from measurements or simulated data from path loss models in coded in programmes like Matlab or data obtained from ray tracing. [5]. All are popular techniques, although ray tracing is becoming more so for the higher frequencies associated with 5G. Delay spread statistical models (such as Saleh-Valenzuela (S-V) [6], shown in Figure 1) use parameters such as cluster arrival time and ray arrival time estimated from measurements or simulations to model the power delay profile (PDP). Deterministic approaches such as ray tracing compute the electric field by evaluating electromagnetic (EM) wave propagation mechanisms for ray paths with respect to the site-specific inventory. In order to ease comparisons, we have dealt with statistics of data from the two methods in the same way. Measurements are needed to create analytical channel models and do not require knowledge of objects in the channel. The ray tracing method uses models based on measurements but does require accurate information about objects in the channel.

In [7]-[18], ray tracing and/or channel measurements were used to obtain site-specific channel model parameters. In [7], [11]-[14], [17], [18], ray tracing was used to obtain large and smallscale parameters such as path gain, Rician K-factor and Root-Mean-Square (rms) delay spread. In [8], [9], large-scale parameters were investigated and [10], [15] considered ray tracing for obtaining small-scale fading effects. Objects in ray tracing models are specified by an approximate representation of the floor plan and the inventory. Consequently, ray tracing models are usually tuned by adjusting material definitions, specifying order of reflections and diffractions 
or by evaluating diffuse scattering [7], [13]. Considering diffuse scattering, the authors in [10] noted that at millimetre wave frequencies, the effect of diffuse scattering in a ray tracing model is negligible. However, in [7], second order diffuse scattering improved the accuracy of the simulation of rms delay spread and received power.

For 3-dimensional (3-D) ray tracing techniques, there is limited literature on use without a detailed floor plan and inventory description. In [19], a physical-statistical approach was used to model a factory floor space by generating random cluster centres and scatterer locations. The scattering objects in this physical-statistical model were defined as finite lossy dielectric cylinders and geometric relation was used to calculate the arrival time of the multipath components (MPCs).

Our hypothesis is that artificial fractal geometries (such as the Sierpinski Square) can be used instead of workshop inventory in ray tracing software packages. By creating a wireless channel with relatively similar time dispersion characteristic, it is envisaged that this methodology will allow ray tracing propagation tools to predict the delay spread. In the literature, fractals have been used for simulating multipath as well estimating the wireless channel [20], [21]. We propose the use of reoccurring object patterns to generate the required shapes in the correct proportions to mimic the workshop floor plan. The mundane task of accurately specifying objects in the ray-tracer has been studied and a generic object placement algorithm for machine workshops of similar dimensions is presented here. By using the exact inventory of two machine workshops (located at the Wolfson School of Mechanical, Electrical and Manufacturing Engineering, Loughborough University and Clemson University [22]), ray tracing models were created and the time dispersion of the wireless channel in both workshops was evaluated in an industry standard ray tracing package (Wireless Insite [23]).

At Loughborough University (Lboro) machine workshop, our channel sounder was used to obtain complex channel frequency responses (CFR) over the frequency range of $2.3-2.5$ $\mathrm{GHz}$ using omnidirectional antennas in a point to multipoint configuration. Adhering to the recommendation in [24], 12 measurements were taken at each receiver location to average out small-scale fading effects. At each of the receiver locations, a uniform rectangular array (URA) with $\lambda / 2$ spacing at the center frequency was used. Taking measurements in workshops that are in use and obtaining accurate inventory representation of workshops contents both presents challenges. In this paper we propose a way to quickly synthesize the inventory of machine workshop spaces using fractal shapes and use ray tracing with that inventory to study delay spread. This combination provides an avenue for adequate radio planning for IoT or $5 \mathrm{G}$ networks. 
The contributions of this paper include:

- A new fractal overlaying algorithm for emulation of machine workshops that allows rapid modelling of RF propagation using ray tracing packages.

- Measured and simulated power delay profiles (PDPs) for a machine workshop.

- Simulated S-V channel parameters at 2.4 and $60 \mathrm{GHz}$ obtained from a machine workshop with typical inventory. The S-V parameters were used to simulate the statistical channel impulse response of the wireless channel.

The remainder of this paper is organized as follows: Section II discusses the VNA method used for our RF measurements and includes a procedure for obtaining the channel frequency response (CFR) and how the ray tracing simulation was created using the measurement scenario. In Section III, we describe the fractal overlaying algorithm and apply it to two workshops of similar dimensions. In Section IV, the process of how to obtain the statistical features of the wideband channel is discussed. In Section V, the simulated delay spread obtained from the fractal object overlaying algorithm and the exact workshop inventory is compared in form of cumulative distribution functions (CDFs). This section also presents the VNA measurements for delay spread at specified receiver locations. In addition, the Saleh-Valenzuela (S-V) model parameters obtained from the fractal overlay ray tracing model is presented as well its prediction ability at $28 \mathrm{GHz}$. In Section VI, we summarize our findings and contributions.

\section{Channel Measurement and Simulation Setup}

\section{A. Measurement Environment}

The diagram in Figure 2 shows the layout of a machine workshop at Loughborough University. This is a light engineering workshop used to support student projects and academic research. The workshop is of medium size with dimensions of $17 \mathrm{~m}$ by $11 \mathrm{~m}$ by $4.5 \mathrm{~m}$. It is located on the ground floor with concrete ceiling. The walls are made of brick and there are metallic enclosures carrying cables from the floor to the roof. The windows were made of glass and the entrance door was made from wood and glass. Within the workshop, metallic equipment such as lathes, drills, workbenches and other machine tools exists and are as listed in Appendix A. Each machine has a walk space around it and its own and shared cabinets for tools and materials. Given that the workshop topography was planned using health and safety regulations common to Europe, it is reasonable to state that there are many other similar machine workshops 


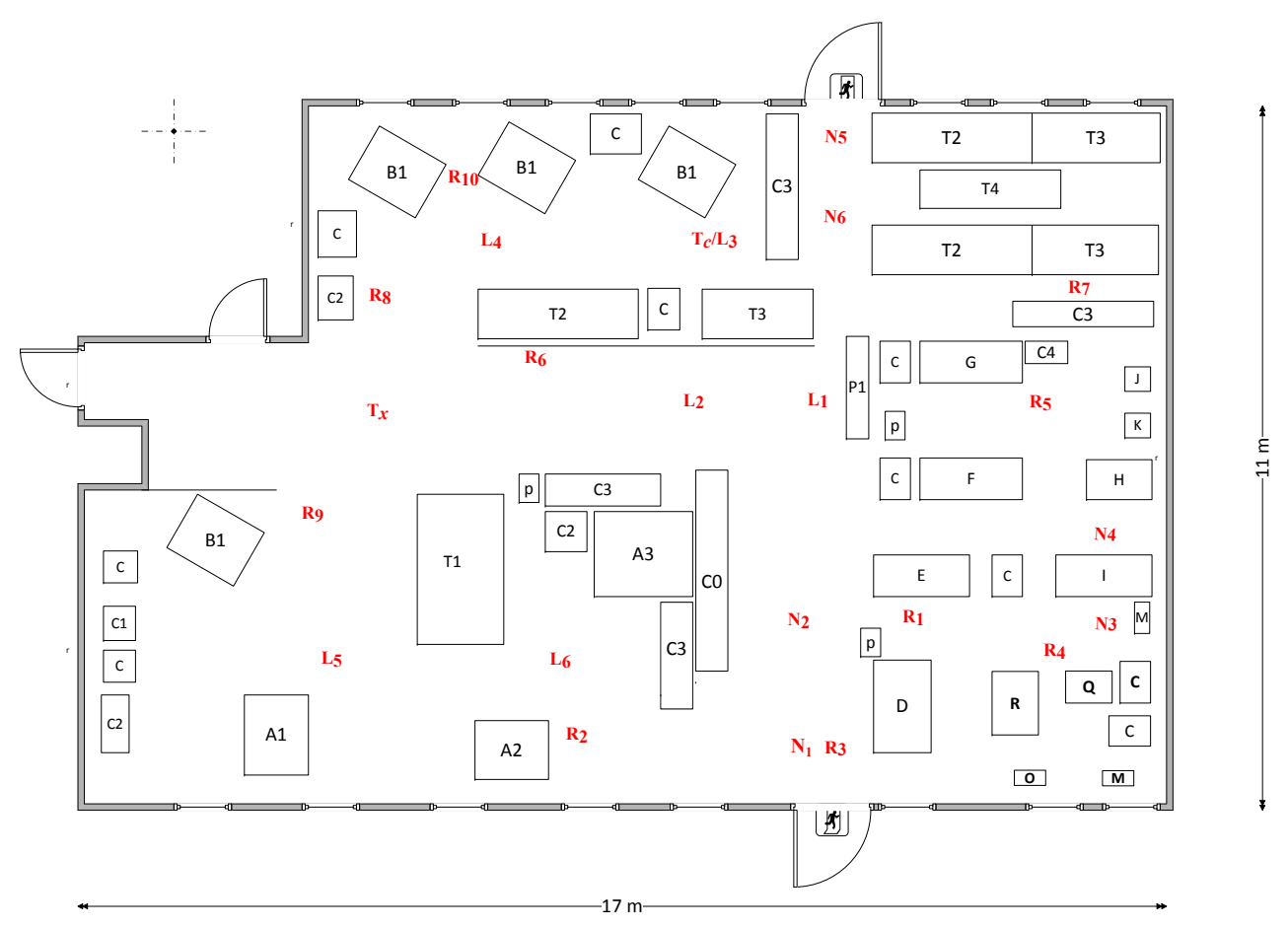

Figure 2. Loughborough University workshop Layout. The plan shows the transmitter and receiver locations. The twelve receiver measurement locations (LoS and NLoS) are represented as $\mathrm{L}_{i}$ and $\mathrm{N}_{i}$. The receiver points $\mathrm{R}_{i}$ and transmitter location $\mathrm{T}_{c}$ were used for computing statistical features of the fractal algorithm. The detailed workshop inventory is included in Appendix A

in existence. In the machine workshop, the receiver locations were classified into non line-ofsight (NLoS) and line-of-sight (LoS) topographies. For LoS topographies, there was a clear and visible direct path between the transmitter and receiver, while NLoS topographies resulted from obstructions created by machine parts, metallic cupboards or pillars.

\section{B. Channel Sounder and Measurement Procedure}

The measurement system was made up of a VNA (Agilent E8050A), two wideband vertically polarised omni directional antennas with $1.2 \mathrm{dBi}$ gain (EM6116), a $13 \mathrm{~m}$ long cable connecting the receiver antenna to port 2 on the VNA and a $3 \mathrm{~m}$ cable connecting the transmitting antenna to port 1 on the VNA. By configuring the VNA in transfer function mode, we transmitted swept signals across $2.3-2.5 \mathrm{GHz}$ from port 1 to port 2 on the VNA. In accordance with the EU exposure limits [25], the equivalent isotropically radiated power (EIRP) was set to $6.21 \mathrm{dBm}$. For the specified frequency span, 1601 frequency points and a sweep time of $800 \mathrm{~ms}$ were used on the VNA. This corresponds to a maximum detectable delay $\left(\tau_{\max }\right)$ of $8 \mu$ s, or a maximum 
path resolution of $2400 \mathrm{~m}$. As a result, the time resolution of the channel sounder is $2 \mathrm{~ns}$. Using a practical inter sensor node distance from [12], the transmitter-receiver $\left(\mathrm{T}_{x} \rightarrow \mathrm{R}_{x}\right)$ separation distance for both NLoS and LoS receiver locations were between 2 and $12 \mathrm{~m}$. Prior to the measurements, the VNA was calibrated using a full 2-port calibration method. The calibration process removed the measurement errors generated by the connectors and cables. The delay versus power relationship of the CFR was obtained by applying an inverse Fourier transform on the CFR. So as to suppress the effect of the band limited Fourier transform, a Kaiser window was applied to the CFR. The CFR obtained from the measurement includes the antenna responses of both the transmitting and receiving antennas. Given that the machine workshop inventory creates a rich multipath environment, it can be assumed that the CFR obtained is independent of the MPC direction [3]. During the measurement campaign, the wireless channel in the workshop was quasi-static. This was achieved by taking the measurements out-of-hours. For this measurement campaign, the transmitter was fixed and paired with twelve receiver locations as shown in Figure 2. Given that the VNA was used for transmitting and receiving simultaneously, the intermediate frequency (IF) bandwidth of the VNA was set to $3 \mathrm{kHz}$ so as to avoid distortion of the received signals.

\section{Ray Tracing Simulation Setup}

In this research the delay spread for the Loughborough University (Lboro) machine workshop was compared with that of [22]. The ray tracing models for both machine workshops were implemented in Wireless Insite. In the ray tracing models, the following material definitions were used: concrete for the floor and ceiling, brick for the walls, glass for the windows and the entrance door. The electromagnetic parameters for the materials in the ray tracing model are presented in Appendix B. These parameters are based on the ITU-R P.2040 [26] recommendation and Wireless Insite material definition. The Loughborough University workshop model also included two metal doors and metal boxes are used for overhead heating, ventilation, and air conditioning (HVAC) pipes and pillars. The simulation settings and parameters for the ray tracing models are summarized in Table I. In both machine workshop models, five transmitters $\left(\mathrm{T}_{x}\right)$ with fifty receivers $\left(\mathrm{R}_{x}\right)$ were used in obtaining the distribution of the rms delay spread. This creates 250 unique $\mathrm{T}_{x} \rightarrow \mathrm{R}_{x}$ combinations on each workshop floor plan. With respect to the prediction accuracy of the ray tracing software, delay spread measurements at $2.4 \mathrm{GHz}$ was used for statistical analysis. In the literature, the accuracy of ray tracing packages is generally regarded 
Table I

RAY TRACING MODEL PARAMETERS

\begin{tabular}{ll}
\hline Parameter & Value \\
\hline Bandwidth & $200 \mathrm{MHz}$ \\
Antenna type & $\lambda / 2$ dipoles \\
Transmitter Antenna gain & $2 \mathrm{dBi}$ \\
Receiver Antenna gain & $2 \mathrm{dBi}$ \\
Transmitter power & $0 \mathrm{dBm}$ \\
Order of reflections & 6 \\
Order of diffractions & 2 \\
Order of transmissions & 2 \\
Antenna polarisation & Vertical \\
\hline
\end{tabular}

as accurate if the mean error and standard deviation of the error are a fraction of the mean value [11].

\section{FRACTAL BASED MODELLing}

Fractals have been used in this research to emulate the scattering properties of inventory inside machine workshops. Fractals are patterns, many of which occur in nature, that can easily be specified mathematically. In artificial fractals, a specified number of self-similar objects are obtained for each fractal level or iteration by recursively evaluating the fractal algorithm. From the physical observation of an engine plant and machine workshops investigated in this work, machine workshop floor plans are populated by few large objects (such as lathes, mills) and many small objects (like machine tool boxes, shelves/cupboards, drawers) with metallic properties. Using this physical floor plan description, the Sierpinski triangle, Cantor set and Sierpinski square fractals were considered for recreating the time dispersion of both machine workshop. Since the large objects present in the workshop possess irregular perimeters and shapes, the fractal object placement algorithm used in this work is based on overlaying fractal objects. This approach creates a pattern of objects with diverse edges and spacing which contribute to creating a rich scattering environment. The fractal object placement algorithm can be summarized in the following steps:

1) Define a square cell using the shorter dimension $\left(L_{s c 1}\right)$ of the machine shop. A cell in this work is defined as a square boundary that imposes space constraints for placing the fractal 
objects.

2) Using the defined square cell, place fractal objects from the first and second fractal iteration/level. These objects can be referred to as "Level 1" and "Level 2" objects.

3) Define a second square cell. This cell is smaller than the cell defined in 1). The length of the second square cell in meters can be expressed as: $L_{s c 2}=L_{s c 1}-1$.

4) Repeat 2) using the cell size defined in 3).

5) Define the smallest square cell with length $L_{s c 3} ; L_{s c 3}=L_{s c 2}-1$.

6) Repeat 2) using the cell size defined in 5).

In Figure 3 a fractal overlay using triangular solids in a Sierpinski square layout is depicted. Using the algorithm described, a machine workshop floor space can be populated with fractal objects of two levels from three different cell sizes. In order to implement this algorithm, the Sierpinski square fractal was used in computing the object spacing for the fractal levels. This reference fractal was selected because its governing rule relies on a square shape. By adjusting the object spacing, the floor plan was split into square cells by a method that is similar to tiling the square. Consequently, the spacing between objects at the same level/iteration were approximated in line with the grid size in the floor plan tool (within Wireless Insite). The length of Level 1 and Level 2 objects in the Sierpinski square fractal overlay was $1 \mathrm{~m}$ and $0.33 \mathrm{~m}$. This was derived from the generic rule for creating the Sierpinski square fractal. With respect to Sierpinski triangle and Cantor set fractal overlays, the object dimensions were inherited from the modified layout generated by the Sierpinski square fractal overlay. The height of all Level 1 objects was set at $3 \mathrm{~m}$ and $2 \mathrm{~m}$ for all Level 2 objects. The fractal object heights used were selected based on the height distribution of the machine workshop inventory. By labelling the fractal objects obtained from overlaying three cells, it becomes possible to create an environment made up of reflective and absorbent materials. This can be achieved by altering the material definition of some of the labelled objects.

\section{FractAl OVERLAY STATISTICAL FEATURES}

Given that large-scale fading effects within an area (of $10 \lambda-40 \lambda$ ) on the machine workshop floor is relatively constant, virtual arrays were adopted in order to evaluate the small-scale fading statistics [27], [28]. The virtual array adopted was made up of a uniform linear array (ULA) of four elements at the transmitter and a uniform rectangular array (URA) of twelve elements (4 $\mathrm{x} 3$ ) at the receiver. This combination gives rise to forty-eight $\mathrm{T}_{x} \rightarrow \mathrm{R}_{x}$ profiles for each 


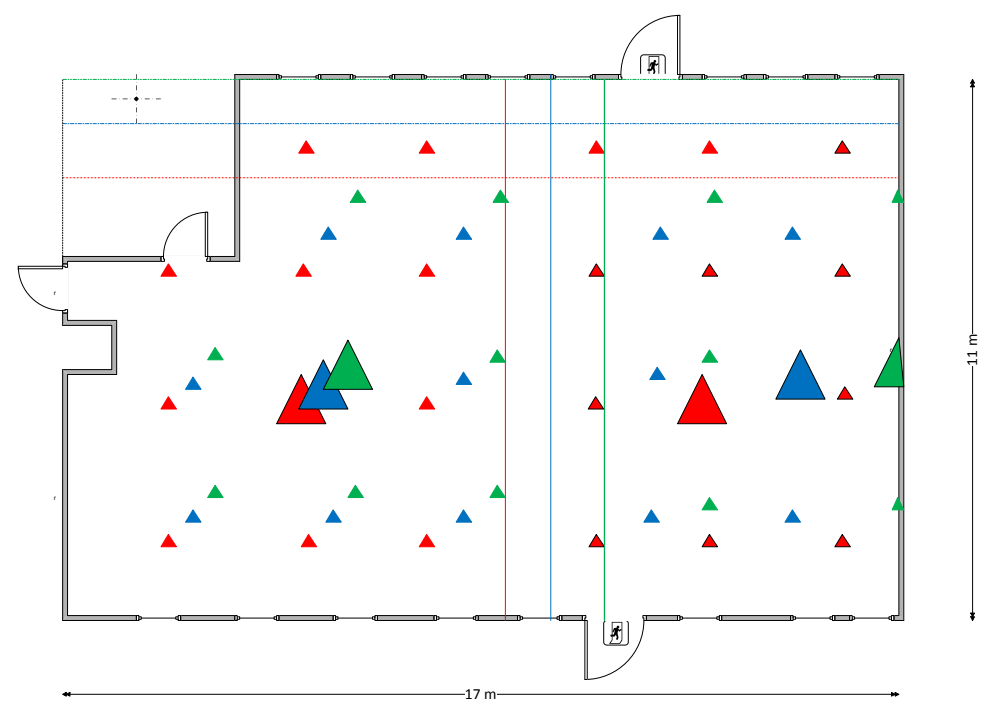

Figure 3. Depiction of the triple cell fractal overlay. The objects in blue are created from the $9 \mathrm{~m}$ cell depicted by red lines, blue objects are from the $10 \mathrm{~m}$ cell and green objects are created from the $11 \mathrm{~m}$ cell

receiver location, which aligns with the requirement presented in [29]. Using the Loughborough University workshop floor plan, S-V parameters were obtained from the simulated virtual array setup. Since both University workshops are of similar dimensions, the fractal overlay for both floor plans are identical. The PDPs were obtained by using one transmitter (located at $\mathrm{L}_{3}$ and ten receiver points $\left(\mathrm{R}_{i}\right)$ placed at $1.5 \mathrm{~m}$ height (in Figure 2). The spacing between each element in both the ULA and URA was $\lambda / 2$. The PDP between the $a^{\text {th }}$ element of the ULA and the $b^{\text {th }}$ element of the URA $P(\tau, a, b)$ shows the power/time distribution between $a$ and $b$. The average PDP (APDP) was obtained by spatially averaging the PDPs such that the first MPC of each profile arrives in the same delay bin for all $P(\tau, a, b)$. With respect to the fractal overlay (on the Loughborough University workshop floor plan), seven of the receiver points were NLoS and three were LoS. The cluster arrival rate $(\Lambda[1 / \mathrm{nsec}])$, ray arrival rate $(\kappa[1 / \mathrm{nsec}])$, cluster decay factor $(\Gamma)$ and ray decay factor $(\gamma)$ which make up the $\mathrm{S}-\mathrm{V}$ model parameters were obtained as follows:

1) Cluster arrival rate: The inter cluster arrival rate $(\Lambda)$ was calculated using the inter cluster arrival time $\left(\Delta T_{l}=T_{l}-T_{l-1}\right) . \Delta T_{l}$ was then averaged for the respective APDP, thus $\Lambda=$ $1 / \overline{\Delta T_{l}}$.

2) Ray arrival rate: The ray arrival rate $(\kappa)$ was obtained based on the recommendations in [27], [30], [31] and the delay bin was selected as $5 \mathrm{~ns}$. 
3) Cluster decay: The cluster decay $(\Gamma)$ for an APDP was obtained by performing linear regression with the normalized power in $\mathrm{dBm}$ and the delay $(\tau)$. The slope of the regression line was then converted to the decay constant by:

$$
\Gamma=\frac{-10}{\ln 10 k_{r e g, l}}
$$

where $k_{\text {reg, } l}$ is the slope of the regression line on $\mathrm{dB}$ scale for cluster $l$.

4) Ray decay: The ray decay constant $(\gamma)$ was calculated in a similar way as the cluster decay constant. For a specified cluster $l$, the ray decay of a cluster was obtained from a linear regression of the logarithmic powers and the delay $(\tau)$ of MPCs within a cluster $l$.

\section{RESULTS AND DisCUSSION}

\section{A. Delay Spread Measurements Versus Ray Tracing}

A $30 \mathrm{~dB}$ threshold was applied to the PDPs obtained from the measurements and the ray tracing software. With this threshold, all MPCs that are $30 \mathrm{~dB}$ less than the highest MPC are discarded in the delay spread calculation. In order to make a fair comparison between the measured delay spread results obtained from the VNA and the predictions made by Wireless Insite, a common maximum excess delay $\left(t_{\eta}\right)$ was applied. The PDP obtained at $L_{1}$ (measurement and simulation) is shown in Figures 4 and 5, where $t_{\eta}$ is selected as $100 \mathrm{~ns}$. The $\tau_{r m s}$ obtained from the VNA measurements and Wireless Insite is presented in Table II and Table III compares the error measures obtained with the literature. In Figure 6, the delay spread is plotted against $\mathrm{T}_{x} \rightarrow \mathrm{R}_{x}$ separation distance. The delay spread increased with distance for both topographies and was generally higher for NLoS sites. This phenomenon has been observed in the literature [3], [12], [31], [32]. The mean error and standard deviation error from the results presented in Table II was 4.33 and 3.36 , which are a fraction of the mean delay spread.

\section{B. Fractal Overlay Delay Spread Distribution}

Using the machine workshop models, $\tau_{r m s}$ was calculated at each of the 250 receiver locations in both workshop models as presented in Section II. In the ray tracing software, the propagation paths were used to determine NLoS and LoS topographies. In Figure 7, the NLoS and LoS CDFs of the simulated delay spread at $2.4 \mathrm{GHz}$ in both machine workshops is compared to the values obtained using the Cantor set and Sierpinski triangle fractal overlays. Due to the characteristics of the Cantor set and Sierpinski triangle fractals, they both generate fractal overlays with sparse 
Table II

COMPARISON OF RMS DELAY SPREAD OBTAINED FROM VNA AND RAY TRACING MODEL

\begin{tabular}{ccc}
\hline \multicolumn{1}{c}{ Path } & VNA (ns) & Wireless Insite (ns) \\
\hline $\mathrm{T} \rightarrow \mathrm{L}_{1}$ & 18.51 & 15.43 \\
$\mathrm{~T} \rightarrow \mathrm{L}_{2}$ & 15.92 & 15.90 \\
$\mathrm{~T} \rightarrow \mathrm{L}_{3}$ & 15.22 & 11.80 \\
$\mathrm{~T} \rightarrow \mathrm{L}_{4}$ & 12.56 & 12.99 \\
$\mathrm{~T} \rightarrow \mathrm{L}_{5}$ & 17.76 & 13.29 \\
$\mathrm{~T} \rightarrow \mathrm{L}_{6}$ & 15.57 & 11.46 \\
$\mathrm{~T} \rightarrow \mathrm{N}_{1}$ & 19.07 & 13.39 \\
$\mathrm{~T} \rightarrow \mathrm{N}_{2}$ & 21.94 & 15.10 \\
$\mathrm{~T} \rightarrow \mathrm{N}_{3}$ & 21.97 & 19.92 \\
$\mathrm{~T} \rightarrow \mathrm{N}_{4}$ & 26.76 & 14.68 \\
$\mathrm{~T} \rightarrow \mathrm{N}_{5}$ & 26.44 & 19.52 \\
$\mathrm{~T} \rightarrow \mathrm{N}_{6}$ & 13.22 & 9.49 \\
\hline
\end{tabular}

Table III

ERROR MEASURE COMPARISON FOR MEASUREMENT AND RAY TRACING PREDICTION

\begin{tabular}{cccc}
\hline Author & Frequency $(\mathrm{GHz})$ & Parameter & MAPE (\%) \\
\hline This work & 2.4 & Delay spread & 22.13 \\
{$[11]$} & 2.4 & Delay spread & 28.39 \\
{$[8]$} & 2.4 & Received power & 57.56 \\
{$[14]$} & 1.3 & Delay spread & 48.81 \\
{$[13]$} & 3.5 & Delay spread & 14.77 \\
{$[10]$} & 60.0 & Delay spread & 20.43 \\
\hline
\end{tabular}

Level 2 objects. Thus they offer little flexibility regarding overlaying objects of different levels or iterations. Additionally, the Cantor set creates LoS topographies for approximately $70 \%$ of the receiver points and the Sierpinski triangle absorbs $55-65 \%$ of the receiver points in its fractal objects. As a result, only the Sierpinski square layout will be discussed further. For the Sierpinski square fractal overlay, two approaches were adopted with respect to the material definition for the fractal objects. In the first run, all the fractal objects were defined as perfect electrical conductors (PEC) and the second with a fraction of the Level 2 objects changed to an absorbent material like wood. The primary purpose of mixing the Level 2 objects was to observe the effect of the material definitions on the EM wave propagation. From the simulations carried 


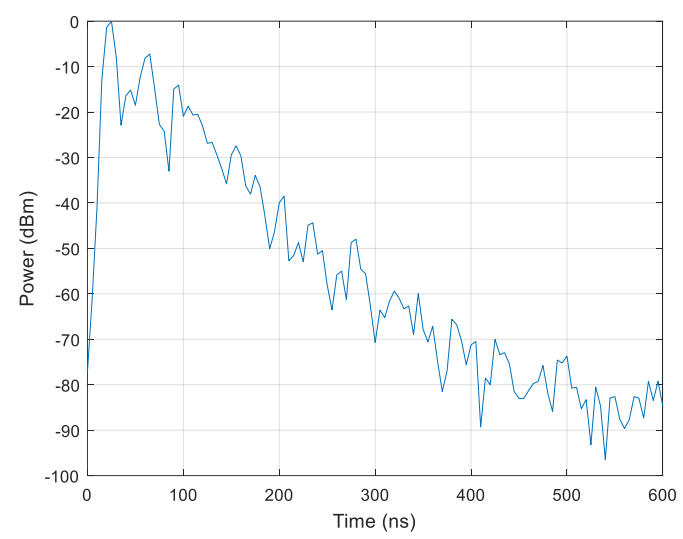

Figure 4. Measurement power delay profile at receiver location $\mathrm{L}_{1}$ for $2.3-2.5 \mathrm{GHz}$. The measurement profile is displayed to $600 \mathrm{~ns}$ in order to illustrate the decaying effect in the MPCs. This exceeds the delay in large factory environments [33]

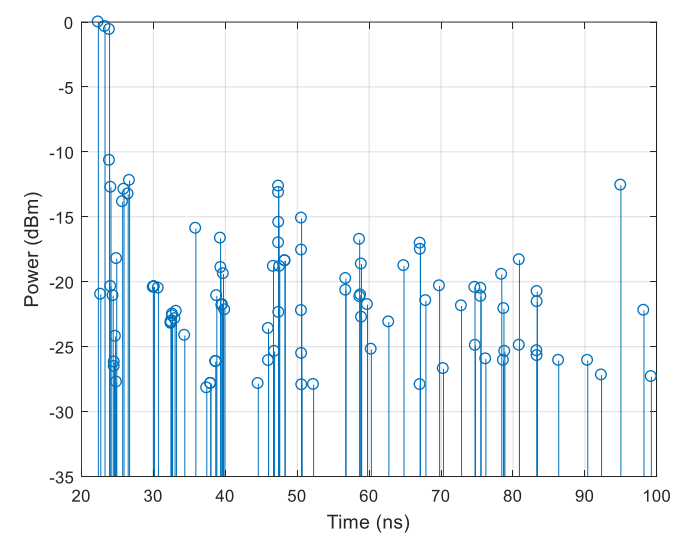

Figure 5. Ray tracing profile at receiver location $\mathrm{L}_{1}$ for $2.3-2.5 \mathrm{GHz}$ showing an excess delay of $100 \mathrm{~ns}$

out, the delay spread for both NLoS and LoS topographies reduced as the amount of wood in the channel increased. This is an expected behaviour, as the environment becomes more absorbent than reflective. Consequently, a non reflecting environment can be easily created by defining the fractal objects as an absorbent material. The fractal mixture used in this work was set at $30 \%$. This percentage was selected as it generated the least prediction error when compared with the exact inventory. In other words, it effectively acts as a compromise between the number of Level 2 objects and the effect of an absorbent material on the delay spread.

With the Sierpinski square fractal overlay, $90 \%$ of the arriving paths were within $15.04 \mathrm{~ns}$ (NLoS) and $10.00 \mathrm{~ns}(\mathrm{LoS})$ in both workshop models at $2.4 \mathrm{GHz}$. Changing the material definition for $30 \%$ of the Level 2 fractal objects to wood caused the MPCs to arrive within 17.78 ns and 


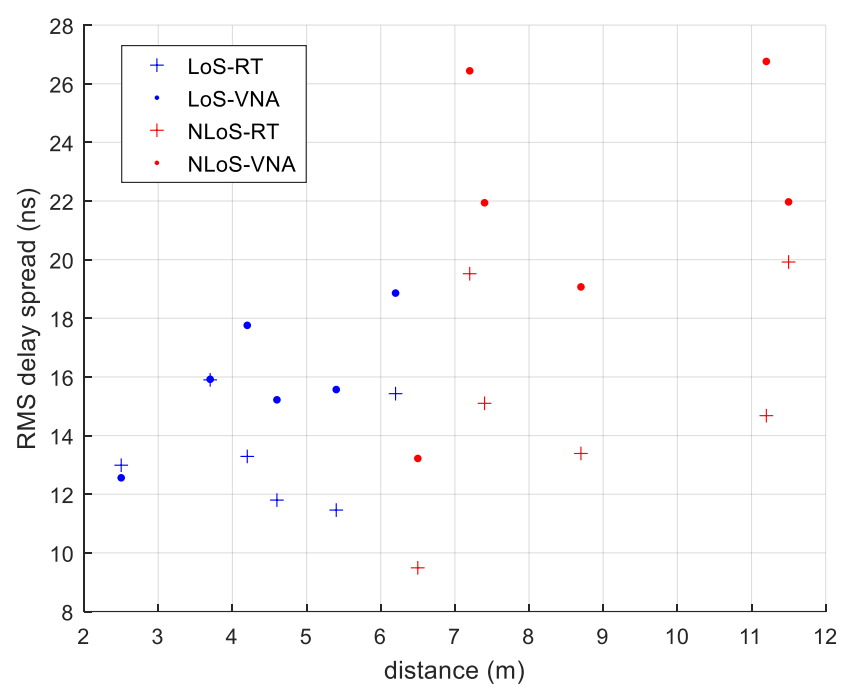

Figure 6. RMS delay spread as a function of distance for all VNA measurements and ray tracing simulation of the exact workshop inventory

$15.00 \mathrm{~ns}$ for NLoS and LoS topographies. At $60 \mathrm{GHz}, 90 \%$ of the arriving NLoS paths were within $15.52 \mathrm{~ns}$ and $15.01 \mathrm{~ns}$ for the fractal overlay mixture. The corresponding LoS values were $12.44 \mathrm{~ns}$ and $11.70 \mathrm{~ns}$. In order to further explore the Sierpinski square layout, the number of edges per $/ \mathrm{m}^{2}$ of the cuboids in the Sierpinski square fractal overlay were reduced to triangular solids. From the simulation runs, it was observed that reducing the number of edges caused the prediction error of the mean delay spread to reduce at both 2.4 and $60 \mathrm{GHz}$. This shows an inverse relationship between the number of edges per $/ \mathrm{m}^{2}$ and the mean delay spread. Thus, the number of edges per $/ \mathrm{m}^{2}$ and the introduction of wooden objects both have dominant effects on the fractal overlay results. With the triangular solids (Sierpinski square) overlay, 90\% of the arriving paths were within $17.98 \mathrm{~ns}$ and $15.78 \mathrm{~ns}$ in both workshop models at $2.4 \mathrm{GHz}$ for NLoS and LoS locations.

When the fractal mixture was adopted, the MPCs arrived within 18.82 ns (NLoS) and 16.60 ns (LoS). At $60 \mathrm{GHz}, 90 \%$ of the arriving NLoS paths were within $17.38 \mathrm{~ns}$ for both the fractal overlay and the its accompanying mixture. The corresponding LoS values were $14.39 \mathrm{~ns}$ and 12.62 ns. The CDF in Figure 8 shows the delay spread at $60 \mathrm{GHz}$ using the triangular solids (Sierpinski square) overlay. This fractal overlay is regarded as Triangular Objects in Table IV. In Table IV, the mean, standard deviation and maximum values of the delay spread obtained from the all fractal overlay scenarios investigated are summarized. From simulation result, the 


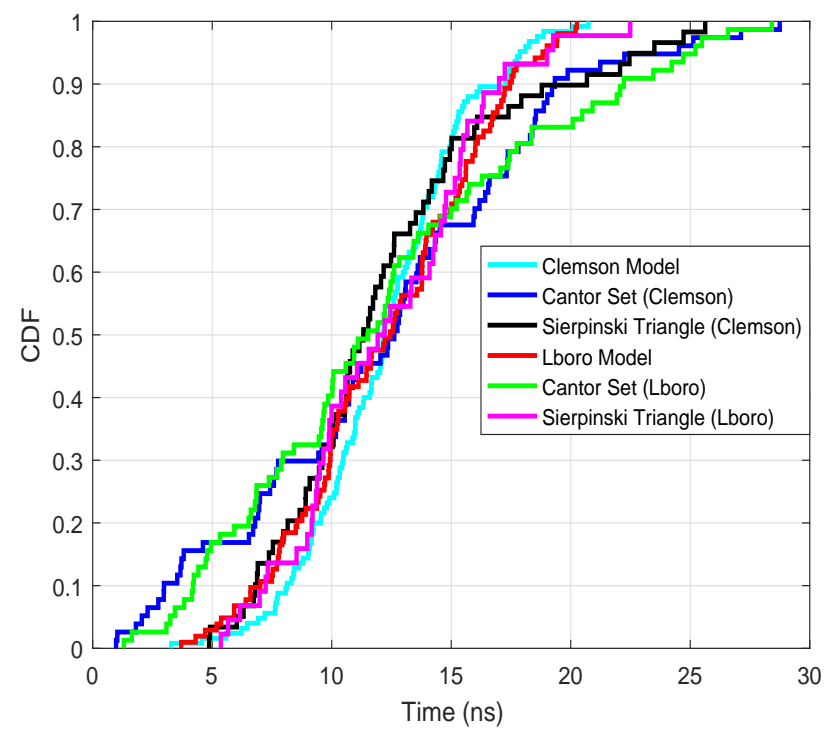

(a)

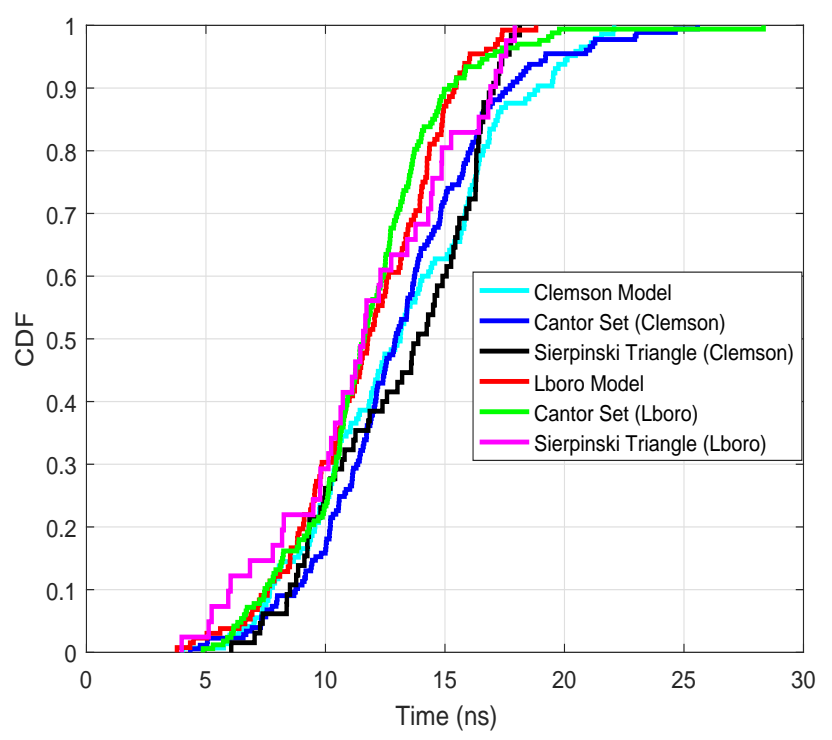

(b)

Figure 7. Simulation of delay spread CDF at $2.4 \mathrm{GHz}$ using Cantor set Fractal overlay and Sierpinski triangle Fractal overlay on both University floor plans a) NLoS and b) LoS

triangular solids (Sierpinski square) overlay reduced the effect of mixing the Level 2 objects with an absorbent material. This overlay choice can be selected when the amount of absorbent material is uncertain. The ability of the fractal model to predict the delay spread at $28 \mathrm{GHz}$ was also investigated and the accompanying CDF is shown in Figure 9.

\section{Validation of Fractal Overlay Model}

From observing Table IV, it can be seen that the triangular objects (Sierpinski square) fractal overlay gives the closest approximation of the time dispersion created by the workshop inventory. This fractal overlay was validated using the extracted S-V model parameters at $2.4 \mathrm{GHz}$. In Table $\mathrm{V}$, a summary of the S-V parameters obtained from the visually identified clusters are presented. In the fractal overlay model, the ray decay $(\gamma)$ was between 0.72 and 20 for NLoS and LoS sites. It also generally increased with the cluster arrival time which aligns with the result in [31]. By applying a manual clustering technique, a total of 38 clusters were investigated. The average cluster number for LoS and NLoS sites at $2.4 \mathrm{GHz}$ was 3 and 4 . At $60 \mathrm{GHz}$, the average cluster number for for both LoS and NLoS sites was 4. Using the UWB impulse response simulation in [30], a $200 \mathrm{MHz}$ filter (2.3 - 2.5 GHz) was applied in order to recreate similar channel conditions. 


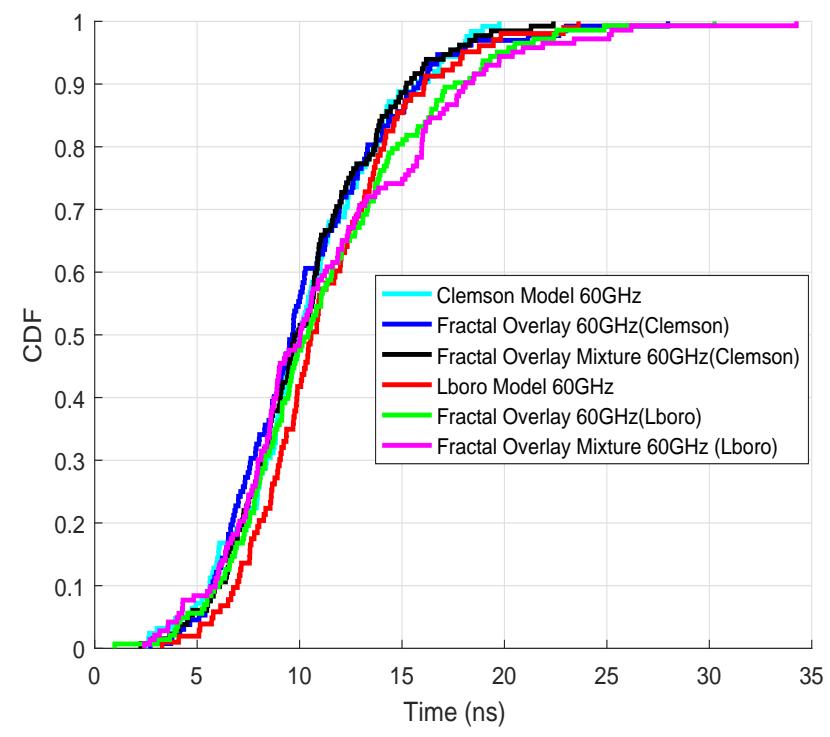

(a)

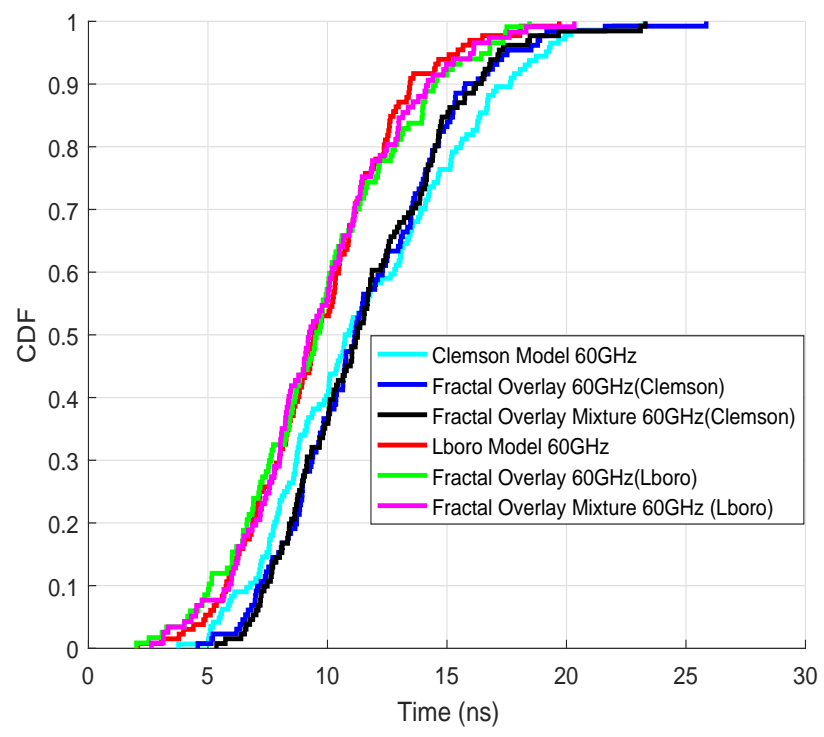

(b)

Figure 8. Simulation of delay spread $\mathrm{CDF}$ at $60 \mathrm{GHz}$ using triangular solids (Sierpinski square) overlay and its mixture on both University floor plans a) NLoS and b) LoS

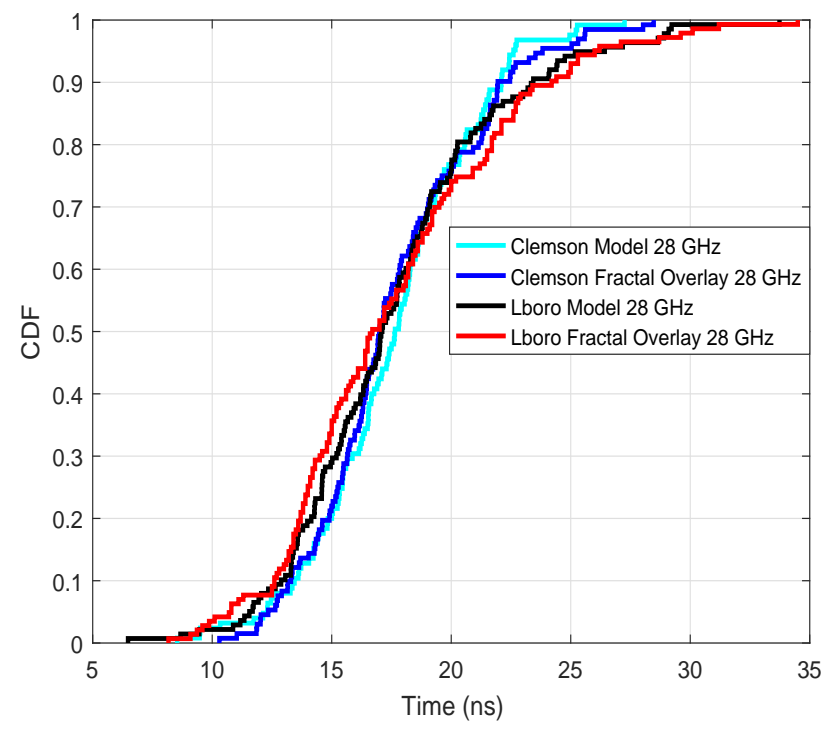

(a)

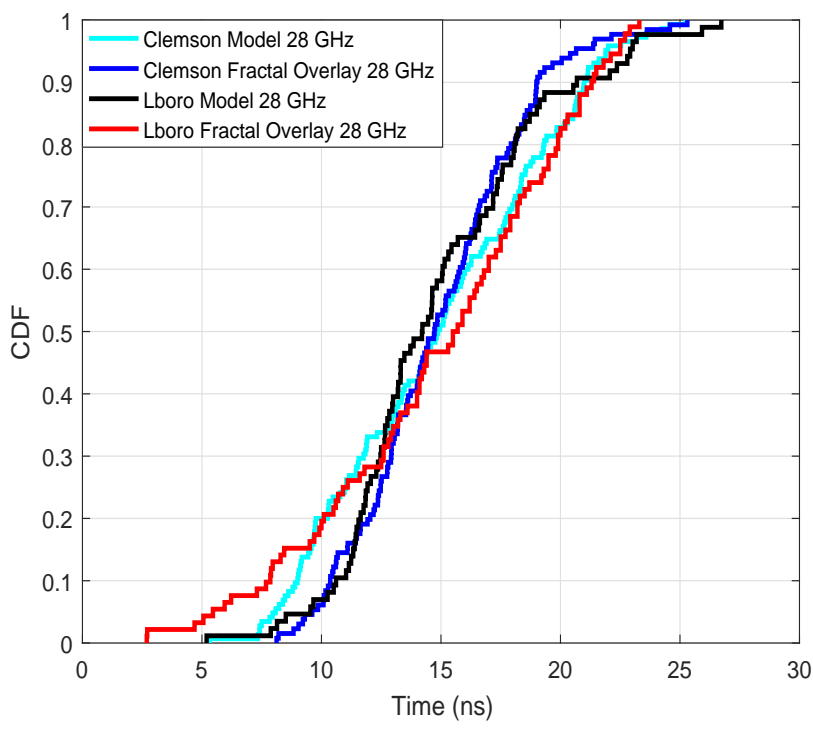

(b)

Figure 9. Simulation of delay spread CDF at $28 \mathrm{GHz}$ using triangular solids (Sierpinski square) overlay on both University floor plans a) NLoS and b) LoS

In Table VI, the delay spread obtained from the bandwidth limited S-V model is compared with 
Table IV

SUMMARY OF MEAN DELAY SPREAD, STANDARD DEVIATION AND MAXIMUM AT 2.4 GHz AND 60 GHz FOR THE FRACTAL OVERLAY COMBINATIONS USED AT CLEMSON UNIVERSITY MODEL AND LOUGHBOROUGH UNIVERSITY WORKSHOP.

\begin{tabular}{|c|c|c|c|c|c|c|c|}
\hline \multirow[b]{2}{*}{ Workshop/ Topography } & \multirow[b]{2}{*}{ Fractal/Model } & \multicolumn{3}{|c|}{$2.4 \mathrm{GHz}$} & \multicolumn{3}{|c|}{$60 \mathrm{GHz}$} \\
\hline & & $\mu(\mathrm{ns})$ & Std. & $\operatorname{Max}(\mathrm{ns})$ & $\mu$ (ns) & Std. & $\operatorname{Max}(\mathrm{ns})$ \\
\hline \multirow{7}{*}{ Clemson/NLoS } & Model & 12.27 & 3.28 & 20.74 & 10.34 & 3.82 & 19.75 \\
\hline & Sierpinski Square (SS) & 9.18 & 3.59 & 22.48 & 9.72 & 4.21 & 24.81 \\
\hline & Triangular objects (TO) & 11.97 & 3.15 & 22.64 & 9.93 & 4.22 & 27.58 \\
\hline & Mixed (SS) & 12.69 & 3.82 & 22.69 & 9.22 & 4.51 & 24.74 \\
\hline & Mixed (TO) & 12.75 & 3.62 & 21.18 & 9.13 & 3.77 & 21.19 \\
\hline & Cantor set & 12.26 & 6.39 & 28.73 & & & \\
\hline & Sierpinski Triangle & 8.43 & 4.88 & 21.79 & & & \\
\hline \multirow{7}{*}{ Clemson/LoS } & Model & 13.16 & 4.10 & 22.08 & 11.61 & 4.20 & 23.26 \\
\hline & Sierpinski Square (SS) & 6.83 & 2.44 & 12.08 & 8.20 & 3.35 & 16.43 \\
\hline & Triangular objects (TO) & 10.12 & 3.08 & 19.33 & 9.15 & 3.60 & 23.39 \\
\hline & Mixed (SS) & 9.94 & 3.72 & 17.42 & 7.23 & 3.21 & 14.25 \\
\hline & Mixed (TO) & 9.63 & 3.51 & 20.40 & 7.87 & 3.47 & 19.56 \\
\hline & Cantor set & 10.42 & 3.72 & 22.84 & & & \\
\hline & Sierpinski Triangle & 10.33 & 3.35 & 15.30 & & & \\
\hline \multirow{7}{*}{ Lboro/NLoS } & Model & 12.28 & 4.00 & 20.25 & 10.24 & 3.83 & 22.62 \\
\hline & Sierpinski Square (SS) & 9.75 & 4.08 & 27.35 & 10.27 & 3.90 & 20.82 \\
\hline & Triangular objects (TO) & 13.31 & 3.69 & 24.26 & 11.23 & 4.78 & 30.26 \\
\hline & Mixed (SS) & 13.28 & 3.54 & 25.17 & 9.41 & 3.96 & 21.73 \\
\hline & Mixed (TO) & 13.14 & 4.17 & 26.21 & 10.52 & 5.37 & 33.54 \\
\hline & Cantor set & 11.75 & 6.64 & 27.87 & & & \\
\hline & Sierpinski Triangle & 6.97 & 3.94 & 17.17 & & & \\
\hline \multirow{7}{*}{ Lboro/LoS } & Model & 11.71 & 3.06 & 18.18 & 9.70 & 3.21 & 19.70 \\
\hline & Sierpinski Square (SS) & 6.35 & 2.35 & 12.37 & 7.69 & 3.11 & 17.92 \\
\hline & Triangular objects (TO) & 11.22 & 3.81 & 19.10 & 9.57 & 3.57 & 18.33 \\
\hline & Mixed (SS) & 9.98 & 3.23 & 17.27 & 6.12 & 2.55 & 13.13 \\
\hline & Mixed (TO) & 11.52 & 4.18 & 20.69 & 8.19 & 3.41 & 18.82 \\
\hline & Cantor set & 10.41 & 3.20 & 27.04 & & & \\
\hline & Sierpinski Triangle & 9.48 & 3.77 & 15.69 & & & \\
\hline
\end{tabular}

the fractal overlay. Upon inspecting the APDPs generated from the fractal overlay model, the following features (which are in agreement with [31]) were observed: 
Table V

Simulated S-V CHANNEL PARAMETERS FROM LOUghbOROUGH UNIVERSITY MACHINE WORKSHOP AT 2.4 GHz

\begin{tabular}{ccc}
\hline S-V parameter & LoS & NLoS \\
\hline$\Gamma$ & 10.90 & 12.83 \\
$\gamma$ & 7.70 & 9.70 \\
$\Lambda$ & 0.04 & 0.06 \\
$\kappa$ & 0.20 & 0.20 \\
\hline
\end{tabular}

Table VI

TRIANGULAR OBJECTS FRACTAL OVERLAY AND S-V MODEL DELAY SPREAD COMPARISON AT 2.4 GHz

\begin{tabular}{ccc}
\hline Scenario & Fractal overlay (ns) & S-V model (ns) \\
\hline LoS & 11.22 & 11.00 \\
NLoS & 13.31 & 14.00 \\
\hline
\end{tabular}

1) Clusters have different decay constants, and the ray decay constant within a cluster increases with delay.

2) Some of the clusters show more than one decay.

3) For all the LoS receiver locations, the first MPC is the strongest and arrives first. After which attenuated MPCs follow. This also occurs with some of the NLoS locations.

Given the number of the receiver locations used in the ray tracing fractal model, a side by side comparison of the fractal overlay delay spread and VNA measurements becomes a statistical challenge due to the population sizes. Since the empirical CDFs obtained from the fractal overlay models resemble a normal distribution, the upper boundary of the average delay spread obtained from the fractal overlay was compared with the measurement results. The mean absolute percentage error obtained from this comparison was $21.17 \%$ for NLoS and $5.61 \%$ for LoS topographies.

\section{CONCLUSION}

In this paper, a method for modelling the delay spread in machine workshops has been developed and validated. This method address the time dispersion characteristic of the wireless channel; a subset of the characteristic features of a wireless propagation modelling. A generic 
ray-tracing package combined with a fractal algorithm was compared with measured results and controls. Delay spread has been studied for NLoS and LoS receiver topographies, measurement campaign results and an equivalent 3-D ray tracing models of two University workshops have been simulated to predict delay spread. The fractal overlay approach for predicting delay spread was validated using accepted statistical wideband channel models and S-V parameters. The effect of altering the material definition of objects obtained from the fractal algorithm iterations was also discussed and shown to reduce the magnitude of the delay spread when more wooden objects were introduced in the wireless channel. From the results presented at higher frequencies, it can be inferred that the method is repeatable. Nonetheless, we recognise that millimetre wave measurements can provide additional data for statistical extrapolation and inference of the model presented. 


\section{APPENDIX A}

\section{MACHINE WORKSHOP INVENTORY}

In Table VII, details of the University machine workshop inventory located at Loughborough University is presented (see Figure 2).

Table VII

WORKSHOP INVENTORY

\begin{tabular}{ll}
\hline Label & Description \\
\hline D & Edwards 3.25mm Truecut \\
E & M300 Harisson \\
F & Colchester Student \\
G & Colchester Triump \\
I & Startite 24-T-1Q \\
H & Metal saw \\
T2,T3 & Wooden shelf 2m high \\
T4 & Wooden table \\
C3 & PEC shelf 2m high \\
M,O & Electrical motors \\
C0 & PEC shelf (2.2m high) with tools \\
B1 & SMX SLV Machine tool \\
A1 & Cut 20P \\
A2 & Haas milling machine \\
C2 & PEC boxes(0.5m high) \\
P & Pillars \\
T1 & Work bench with tools \\
\hline
\end{tabular}




\section{APPENDIX B}

\section{Electromagnetic Material Parameters}

In Table VIII, the permittivity and conductivity of the materials used in the ray tracing models are presented.

Table VIII

PeRmitTIVITY \& CONDUCtivity of RAY TRACING MATERIALS

\begin{tabular}{cccc}
\hline Material & Frequency (GHz) & Permittivity & Conductivity \\
\hline \multirow{3}{*}{ Wood } & 2.4 & 5.00 & 0.0000 \\
& 28 & 1.99 & 0.1672 \\
& 60 & 1.99 & 0.3874 \\
\hline \multirow{2}{*}{ Glass } & 2.4 & 2.40 & 0.0000 \\
& 28 & 6.27 & 0.2287 \\
& 60 & 6.27 & 0.5674 \\
\hline \multirow{2}{*}{ Concrete } & 2.4 & 7.00 & 0.0150 \\
& 60 & 5.31 & 0.4838 \\
Brick & & 5.31 & 0.8967 \\
\hline
\end{tabular}




\section{REFERENCES}

[1] G. Trade and Invest, "Industrie 4.0, Smart Manufacturing for the Future." [Online]. Available: https://www.gtai.de

[2] M. Centenaro, L. Vangelista, A. Zanella, and M. Zorzi, "Long-range communications in unlicensed bands: the rising stars in the iot and smart city scenarios," IEEE Wireless Communications, vol. 23, no. 5, pp. 60-67, October 2016.

[3] S. Chiu, J. Chuang, and D. G. Michelson, "Characterization of UWB channel impulse responses within the passenger cabin of a boeing 737-200 aircraft," IEEE Transactions on Antennas and Propagation, vol. 58, no. 3, pp. 935-945, March 2010.

[4] T. Rappaport, Wireless Communications: Principles and Practice, ser. Prentice Hall communications engineering and emerging technologies series. Prentice Hall PTR, 2002. [Online]. Available: https://books.google.co.uk/books?id=TbgQAQAAMAAJ

[5] K. Pahlavan and A. Levesque, Wireless Information Networks, ser. Wiley Series in Telecommunications and Signal Processing. Wiley, 2005. [Online]. Available: https://books.google.co.uk/books?id=6vhSAAAAMAAJ

[6] A. A. M. Saleh and R. Valenzuela, “A statistical model for indoor multipath propagation," IEEE Journal on Selected Areas in Communications, vol. 5, no. 2, pp. 128-137, February 1987.

[7] M. T. Martinez-Ingles, D. P. Gaillot, J. Pascual-Garcia, J. M. Molina-Garcia-Pardo, M. Lienard, and J. V. Rodríguez, “Deterministic and experimental indoor mmw channel modeling," IEEE Antennas and Wireless Propagation Letters, vol. 13, pp. 1047-1050, 2014.

[8] J. H. Jung, J. Lee, J. H. Lee, Y. H. Kim, and S. C. Kim, "Ray-tracing-aided modeling of user-shadowing effects in indoor wireless channels," IEEE Transactions on Antennas and Propagation, vol. 62, no. 6, pp. 3412-3416, June 2014.

[9] R. Felbecker, W. Keusgen, and M. Peter, "Incabin millimeter wave propagation simulation in a wide-bodied aircraft using ray-tracing," in 2008 IEEE 68th Vehicular Technology Conference, Sept 2008, pp. 1-5.

[10] M. Peter, W. Keusgen, and R. Felbecker, "Measurement and ray-tracing simulation of the $60 \mathrm{GHz}$ indoor broadband channel: Model accuracy and parameterization," in The Second European Conference on Antennas and Propagation, EuCAP 2007, Nov 2007, pp. 1-8.

[11] F. Fuschini, H. El-Sallabi, V. Degli-Esposti, L. Vuokko, D. Guiducci, and P. Vainikainen, “Analysis of multipath propagation in urban environment through multidimensional measurements and advanced ray tracing simulation," IEEE Transactions on Antennas and Propagation, vol. 56, no. 3, pp. 848-857, March 2008.

[12] X. H. Mao and Y. H. Lee, "UHF propagation along a cargo hold on board a merchant ship," IEEE Transactions on Wireless Communications, vol. 12, no. 1, pp. 22-30, January 2013.

[13] A. O. Kaya, L. J. Greenstein, and W. Trappe, "Characterizing indoor wireless channels via ray tracing combined with stochastic modeling," IEEE Transactions on Wireless Communications, vol. 8, no. 8, pp. 4165-4175, August 2009.

[14] S. Y. Seidel and T. S. Rappaport, "A ray tracing technique to predict path loss and delay spread inside buildings," in Global Telecommunications Conference, 1992. Conference Record., GLOBECOM '92. Communication for Global Users., IEEE, Dec 1992, pp. 649-653 vol.2.

[15] A. Karstensen, W. Fan, I. Carton, and G. F. Pedersen, "Comparison of ray tracing simulations and channel measurements at mmwave bands for indoor scenarios," in 2016 10th European Conference on Antennas and Propagation (EuCAP), April 2016, pp. 1-5.

[16] C. Ling, X. Yin, H. Wang, and R. S. Thomä, "Experimental Characterization and Multipath Cluster Modeling for 13 17 GHz Indoor Propagation Channels," IEEE Transactions on Antennas and Propagation, vol. 65, no. 12, pp. 6549-6561, Dec 2017. 
[17] T. A. Thomas, H. C. Nguyen, G. R. MacCartney, and T. S. Rappaport, “3D mmwave channel model proposal," in 2014 IEEE 80th Vehicular Technology Conference (VTC2014-Fall), Sept 2014, pp. 1-6.

[18] S. Hur, S. Baek, B. Kim, J. Park, A. F. Molisch, K. Haneda, and M. Peter, "28 GHz channel modeling using 3D ray-tracing in urban environments," in 2015 9th European Conference on Antennas and Propagation (EuCAP), May 2015, pp. 1-5.

[19] M. Cheffena, "Industrial indoor multipath propagation : A physical-statistical approach," in IEEE 25th Annual International Symposium on Personal, Indoor, and Mobile Radio Communication (PIMRC), Sept 2014, pp. 68-72.

[20] G. Hu, L. Zhang, G. Bi, and S. Zhu, "Fractal based channel estimation for wcdma systems," in IEEE 60th Vehicular Technology Conference, 2004. VTC2004-Fall. 2004, vol. 2, Sep. 2004, pp. 880-884 Vol. 2.

[21] L. Sun, C. Fu, and Z. Zhang, "A fractal-based simulation scheme for multipath fading channel," in 2007 International Conference on Wireless Communications, Networking and Mobile Computing, Sep. 2007, pp. 1060-1063.

[22] L. Tang, K. C. Wang, Y. Huang, and F. Gu, "Channel characterization and link quality assessment of IEEE 802.15.4compliant radio for factory environments," IEEE Transactions on Industrial Informatics, vol. 3, no. 2, pp. 99-110, May 2007.

[23] Remcom, "Wireless InSite Propagation Software." [Online]. Available: https://www.remcom.com/wireless-insite-empropagation-software/

[24] C. W. Kim, X. Sun, L. C. Chiam, B. Kannan, F. P. S. Chin, and H. K. Garg, "Characterization of ultra-wideband channels for outdoor office environment," in IEEE Wireless Communications and Networking Conference, 2005, vol. 2, March 2005, pp. 950-955 Vol. 2.

[25] Working Group "Frequency Management", "ECC RECOMMENDATION (02)04 (revised Bratislava 2003, Helsinki 2007)," Tech. Rep. 02, 2007.

[26] I.-R. R. P.2040-1, "Effects of building materials and structures on radiowave propagation above about $100 \mathrm{mhz}$," Tech. Rep., 2013. [Online]. Available: https://www.itu.int/rec/R-REC-P.2040-1-201507-I/en

[27] E. Tanghe, W. Joseph, J. D. Bruyne, L. Verloock, and L. Martens, "The industrial indoor channel: Statistical analysis of the power delay profile," AEU - International Journal of Electronics and Communications, vol. 64, no. 9, pp. 806 - 812, 2010. [Online]. Available: http://www.sciencedirect.com/science/article/pii/S1434841109001848

[28] A. F. Molisch, "Ultra-wide-band propagation channels," Proceedings of the IEEE, vol. 97, no. 2, pp. 353-371, Feb 2009.

[29] A. F. Molisch, K. Balakrishnan, C.-C. Chong, S. Emami, A. Fort, J. Karedal, J. Kunisch, H. Schantz, U. Schuster, and K. Siwiak, "IEEE 802.15. 4a channel model-final report,” IEEE P802, vol. 15, no. 04, p. 0662, 2004.

[30] A. F. Molisch, J. R. Foerster, and M. Pendergrass, "Channel models for ultrawideband personal area networks," IEEE Wireless Communications, vol. 10, no. 6, pp. 14-21, Dec 2003.

[31] J. Karedal, S. Wyne, P. Almers, F. Tufvesson, and A. F. Molisch, “A Measurement-Based Statistical Model for Industrial Ultra-Wideband Channels," IEEE Transactions on Wireless Communications, vol. 6, no. 8, pp. 3028-3037, August 2007.

[32] M. Boutin, A. Benzakour, C. L. Despins, and S. Affes, "Radio wave characterization and modeling in underground mine tunnels," IEEE Transactions on Antennas and Propagation, vol. 56, no. 2, pp. 540-549, Feb 2008.

[33] T. S. Rappaport, "Characterization of UHF Multipath Radio Channels in Factory Buildings," IEEE Transactions on Antennas and Propagation, vol. 37, no. 8, pp. 1058-1069, 1989. 